\title{
ADVANTAGES OF NANOSENSORS IN THE DEVELOPMENT OF INTERFACES FOR BIOELECTRIC PROSTHESES
}

\author{
Diana Avdeeva ${ }^{1 *}$, Ekaterina Zakharchenko ${ }^{1}$, Dang Quang Nguyen ${ }^{1}$, Nikita Turushev ${ }^{1}$, \\ Aryslan Abdrakhmanov ${ }^{1}$, Roman Vorona ${ }^{1}$, Arnold Popkov $^{2}$, Sergey Tverdokhlebov ${ }^{1}$ \\ ${ }^{1}$ National Research Tomsk Polytechnic University, 634050, Tomsk, Russia \\ ${ }^{2}$ Federal State-Financed Institution "Russian Ilizarov Scientific Center for Restorative Traumatology \\ and Orthopaedics of the RF Ministry of healthcare", 640014, Kurgan, Russia
}

\begin{abstract}
The present research aims to explore the bioelectric activity of muscles using a high-resolution electromyograph and to analyze the prospects of the electromyograph to develop bioelectric patterns for the prosthesis control method based on the data recognition system. The activity of the healthy forearm muscles was investigated during the cyclic activity of fingers in different modes. In addition, the impact of filters on the quality and informativity of myoelectric signals, as well as on the development of bioelectric activity patterns was analyzed. The virtually developed bandpass filters were utilized as experimental filters. The filter impact analysis included the comparison of the signal recorded in the frequency band from 0 to $10000 \mathrm{~Hz}$ with the signal filtered in the frequency band from 20 to $500 \mathrm{~Hz}$. The research revealed the advantages of a high-resolution electromyogram for the pattern recognition-based myocontrol.
\end{abstract}

\section{Introduction}

Loss of a limb as a result of accident or injury can have a considerable psychological and physical impact. The upper limb amputees experience difficulties with simple thing that do not usually require any efforts. In such cases, prostheses allow people to return to a near normal life: to work, to do sport, to cook, etc.

There are three most common types of hand prosthetic devices: cosmetic, bodypowered, and bioelectric prostheses.

Cosmetic hand prostheses are designed to hide the lack of a limb from stares in public. Natural-looking prostheses are focused on the aesthetic appearance, not on the restoration of motor functions. This type of prosthesis has been used to restore a partially amputated hand (Figure 1,a) [1].

The first active upper-limb gripping hook prosthesis was developed by Dorrance in 1912. The first version of this prosthesis was limited to gripping and holding objects [2].

\footnotetext{
*Corresponding author: diana.avdeeva@mail.ru
} 
The device was a body-powered prosthesis. The executing mechanism of the device was a hook with a pinching mechanism to perform motions by the system of belts and a spring. Movements of the patient's healthy upper arm with one of the expandable prosthesis belts attached were used as control information. The patient had to pull the unclamping belt by a healthy part of the arm to open the clamping mechanism of the prosthesis. The belt pulled back the ends of the clamping mechanism to open the prosthesis. Modern body-powered prostheses function in a similar way. The main disadvantages of the mechanical prosthetic hands are as follows: short service life; limited power grasping; a limited number of degrees of freedom; linkage system can cause discomfort and limit activities; unsightly appearance [1].

The development of science and technology allowed scientists to create prostheses which can be controlled by the registration of bioelectric potentials of a human body. These prostheses are bioelectric (Figure 1,b). Bioelectric prostheses perform the function of hand rotation, capturing and holding objects. The main advantage of bioelectric prostheses is a high grasping power and a large number of degrees of freedom. Due to its unique functions, the bioelectric prosthesis allows disabled people to cope with small objects such as a ballpoint pen, fork, spoon, etc. But this type of prosthesis has its own disadvantages: need for maintenance; higher cost and increased weight compared to other types of prostheses; need for the battery charge [1].

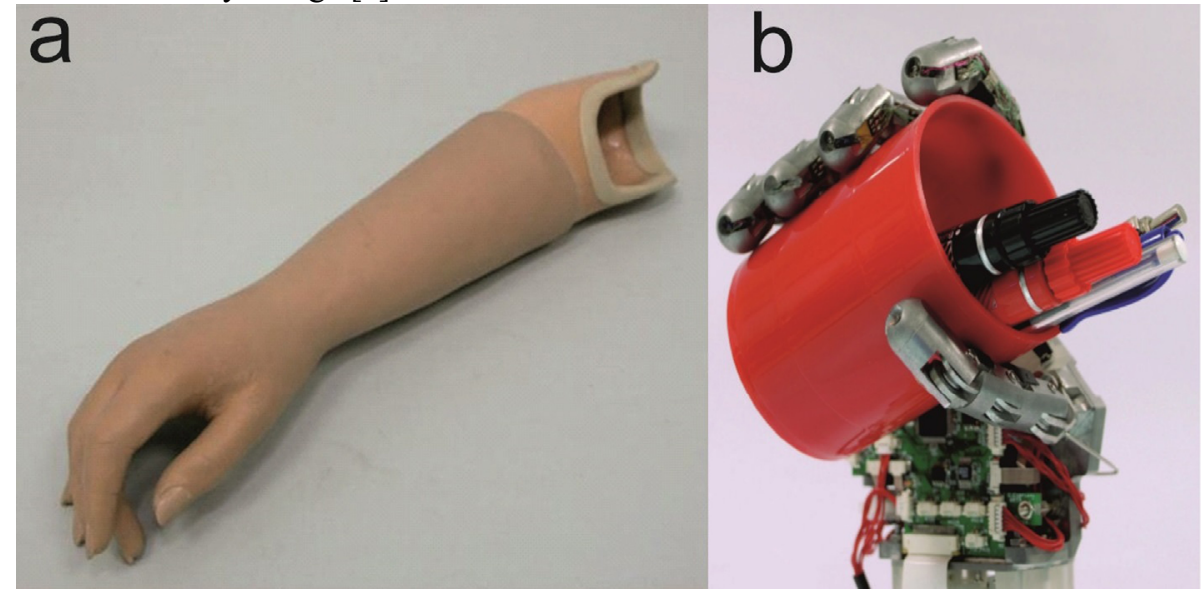

Figure 1. Types of prostheses: $\mathrm{a}$ - cosmetic [3], b- bioelectric [4].

Modern bioelectric prostheses are usually controlled by one of the three main control methods: brain-computer interface, neuroelectric, and electromyographic (myoelectric) [5].

The brain-computer interface method uses a subcortical signal as the primary data source. The signal is recorded using the leads implanted into a brain cortex. The obtained data are processed and transmitted to the mechanical part of prosthesis [6]. Prostheses with this type of control are used by people suffering from paralysis of limbs caused by violation of neural connections between the brain and the corresponding organ (tetraplegia, demyelinization of nerve channels). The main disadvantage of the method is its invasiveness, which can cause the infection or injury of the brain during the implantation of electrodes and the prosthesis operation [5].

The neuroelectric method of control uses signals recorded from the active motor nerves of a person. Electrodes can be implanted in nervous tissue (regenerative, an electrode cuff) or placed over the nerves [6]. The main advantages of neuroelectric method are as follows: the possibility of sensitivity restoration by electric stimulation of the sensory nerve fibers and the lack of dependence on the muscles of a missing limb [7]. The disadvantages of the method include the following: invasiveness; low amplitude of the informative signal in 
comparison with other sources of bioelectric potentials; necessity to design a percutaneous interface; inability to be used in completely paralyzed patients. The main risk of using such prostheses is the possibility of destruction or damage of the nerve and surrounding tissues, and postoperative infections [5].

The electromyographic method uses a myoelectric signal as the primary data source to control prostheses. Myoelectric signal is a signal that is recorded from the excited muscle fibers. This method of prosthesis control consists in registration of the muscle electrical activity signal using surface electrodes. Then the signal is processed by the input amplifier circuits and converted into a digital code. The digital code is analyzed by the microcontroller of the control unit and converted into a command for the prosthesis executing mechanism (Figure 2) [8].

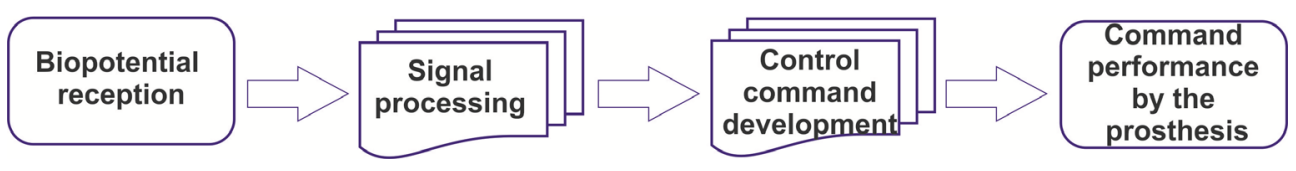

Figure 2. Flow chart of the prosthesis control system.

The main advantages of the electromyographic control method are its non-invasiveness and simplicity of the prosthesis control. The result of the prosthesis operation using this method depends on several factors: the number of informative areas, the arrangement of electrodes on the informative surface, changes in the position of electrodes during operation. The use of prostheses using electromyographic control method requires activity and integrity of the muscle fiber, which is responsible for control of the missing limb [9].

One of the examples of a modern myoelectric prosthesis is a bioelectric prosthesis SmartHand (Figure 1, $b$ ). This prosthesis was developed by researchers of Lund University in Sweden and Scuola Superiore Sant Anna in Italy. This device comprises built-in touch sensors on the prosthesis artificial fingers, which are capable of partial restoration of touch perception [4].

Prosthetics falls behind in technological development in spite of the advances in this branch of surgery and organization of prosthesis control. A number of problems in prosthetics remain unsolved [10]. The amputees experience difficulties with significant weight of prostheses; anatomical inequality of the prosthesis and a healthy limb; necessity to make additional efforts which exhaust a patient. All these disadvantages bring discomfort for patients and force them to think about prosthesis. The study of mechanisms of a healthy limb functioning, bioelectric processes in healthy limbs and their interaction will help to determine the appropriate set of bioelectric signal characteristics for the qualitative control of prosthesis.

For example, the determination of specific characteristics of the active muscle myoelectric signal enables organization of the prosthesis control, which uses the patterns of harmonic sets of signal spectrum. This provides the control system with additional functions with minimum number of leads. The organization of this type of control requires myographic investigation of bioelectrical activity of target muscles to control the prosthesis. The quality of the obtained data depends on sensitivity and noise immunity of diagnosing devices.

\section{Materials and methods}

The research aims to study bioelectric activity of muscles of a healthy limb, namely the forearm, in order to define electrically active zones, the dependence of bioelectric activity of muscles on the geometric position of electrodes in space using a high resolution 
electromyograph, and the impact of filters on the waveform. The nanosensor-based electromyograph utilized in the research enables measurement of the electrical activity of muscles within the frequency range from 0 to $10,000 \mathrm{~Hz}$ with a minimal sensitivity threshold of potential $(0.3 \mu \mathrm{V})$ without standard filter circuits [5, 6]. A high level of noise immunity is due to nanosensors, which eliminate most adverse effects of electrodes, such as polarization during operation and dryout of contact electrolytes as a result of a longduration use [11]. The exclusion of filters from the measuring circuit allows us to eliminate the impact of filters on the amplitude and phase characteristics of the measuring signal and to increase the rate of data processing.

The practical part of the research consisted in the registration of myographic potentials from different positions on the forearm during the repetitive isolated finger motions. Three types of repetitive exercises were performed during the conducted experiments: fingers at rest, weak slow finger flexion, and intensive finger flexion with tension. Psychosomatic response of skeletal muscles to changes in ambience was also considered. For this purpose, the patient was asked to close his eyes for a short period of time during the first phase of experiment. The exercises were applied to three main fingers playing a key role in the hand functioning: a thumb, point finger, and middle finger. Bioelectric signals were obtained from the forearm surface using a bipolar configuration of nanosensors with a common reference wire. The sensors for upper-limb prostheses were placed on the electrically active zones, selected according to Vorotnikov's study of dispersal of bioelectric potentials (Figure 3) [9].

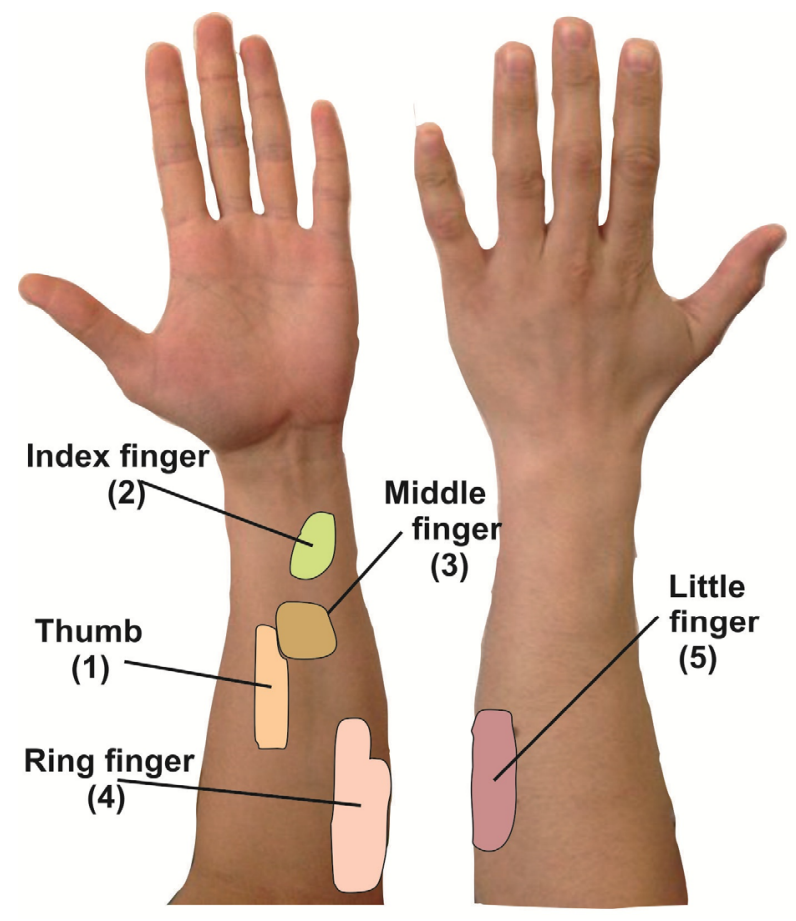

Figure 3. Location of bioelectrical activity zones on the forearm according to S.A. Vorotnikov.

The obtained broadband signals were processed using a bandpass digital filter with cutoff frequencies 20 and $500 \mathrm{~Hz}$, that correspond to the standard range of informative electromyographic signals [12]. 


\section{Results and discussion}

The obtained results are considered through the example of bioelectric activity of the point finger at rest and in the state of active finger flexion and extension. Figure 4 shows electromyograms of the rest state (Figure 4, $a$ ), brain-computer interface (Figure 4, b), weak finger activity (Figure 4,c), and intensive finger activity (Figure 4, $d$ ) obtained by a highresolution electromyograph without filters.

a)

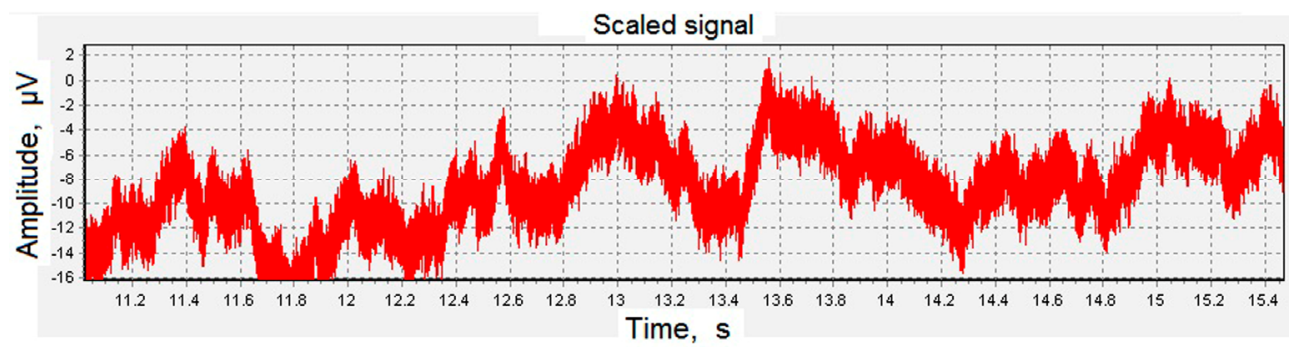

b)

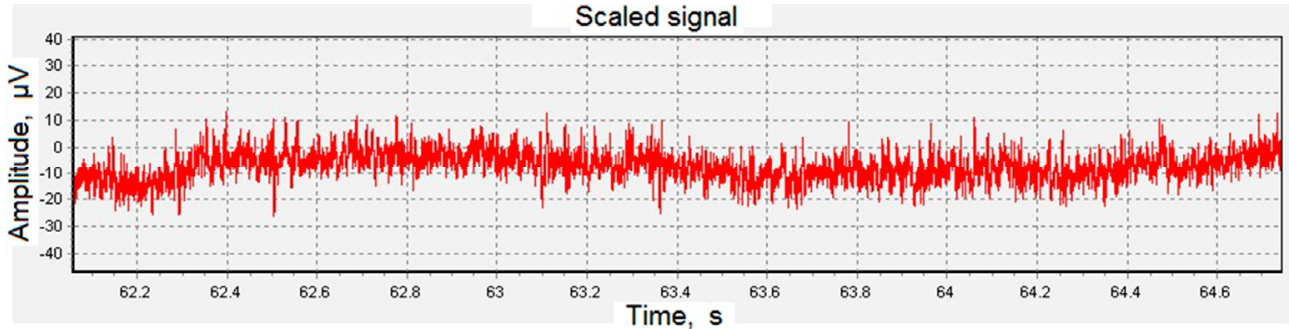

c)

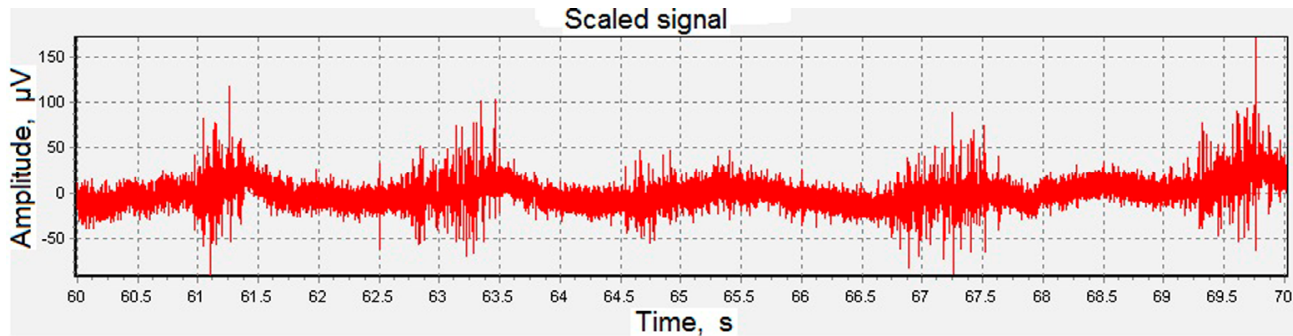

d)

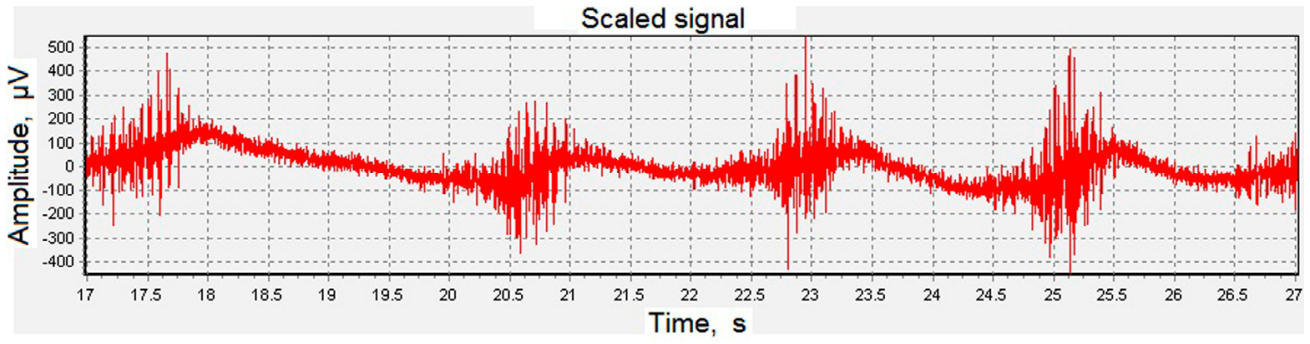

Figure 4. Electromyogram of the point finger activity: a - rest state in the band from 0 to $10,000 \mathrm{~Hz}$; $\mathrm{b}$ - brain-computer interface in the band from 0 to $10,000 \mathrm{~Hz} ; \mathrm{c}-$ weak finger flexions in the band from 0 to $10,000 \mathrm{~Hz}$; $\mathrm{d}$ - intensive finger flexions in the band from 0 to $10,000 \mathrm{~Hz}$. 
The analysis of the rest state electromyogram revealed that at the moment when a patient closed his eyes, the muscle psychosomatic response to changes in visual perception and mental state was registered as a sharp increase of the electromyographic signal direct component on $140 \mu \mathrm{V}$ (Figure 5).

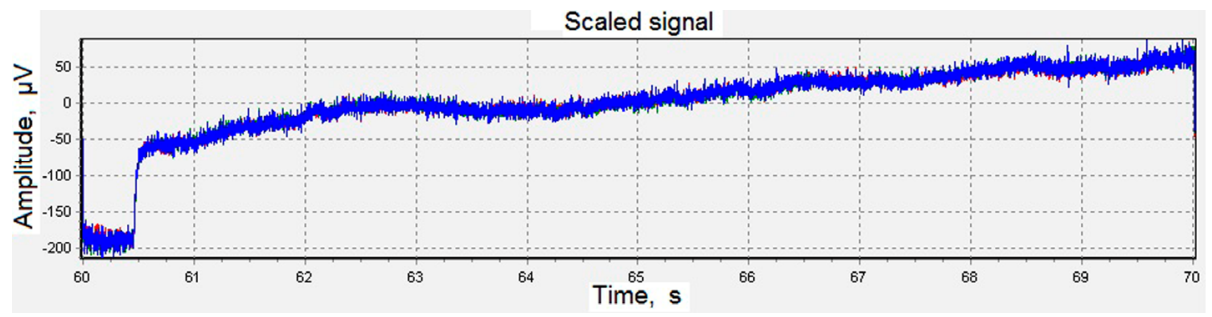

Figure 5. A sharp increase of the direct component in the rest state after closing the eyes.

The analysis of the weak and intensive flexion electromyograms confirmed that bioelectric activity zones of fingers located on the forearm, which were proposed by Vorotnikov, correspond to those in [3]. Thus, significant changes in bioelectric potential level occur periodically during the activity of a certain finger.

The obtained results allow us to assume that cyclically repeating fluctuations of a highresolution myoelectric signal, which change simultaneously with the finger movements, can be applied for the development of a bioelectric control system based on the detection of potential threshold values and data recognition techniques for specific signal harmonics. For example, low-frequency components contain information not only about the muscle volume alteration, but about the muscle fatigue, the current state of the nervous system, etc. This enables the creation of new systems to control prosthetic devices and evaluate the user's state in a real-time mode. In addition, the systems will be able to perform preventive actions in case of changes in patient condition.

The analysis of the filtered and initial electromyograms of intensive flexions of the point finger revealed significant changes in the waveform and decrease in the signal level.

a)

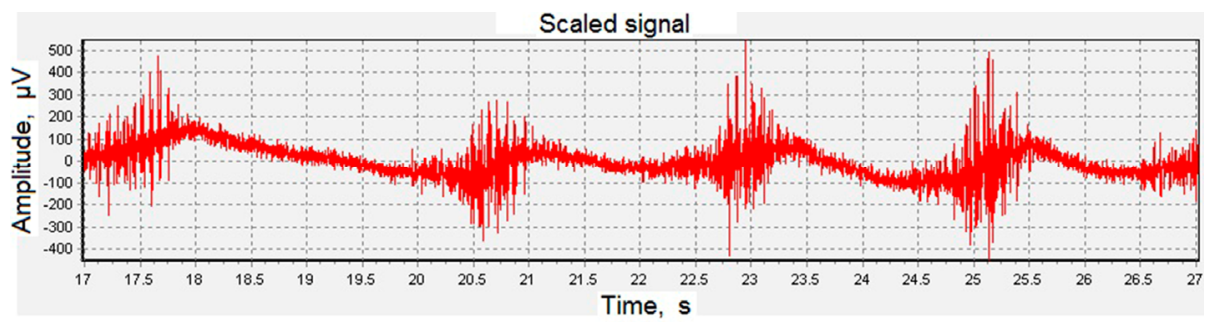

b)

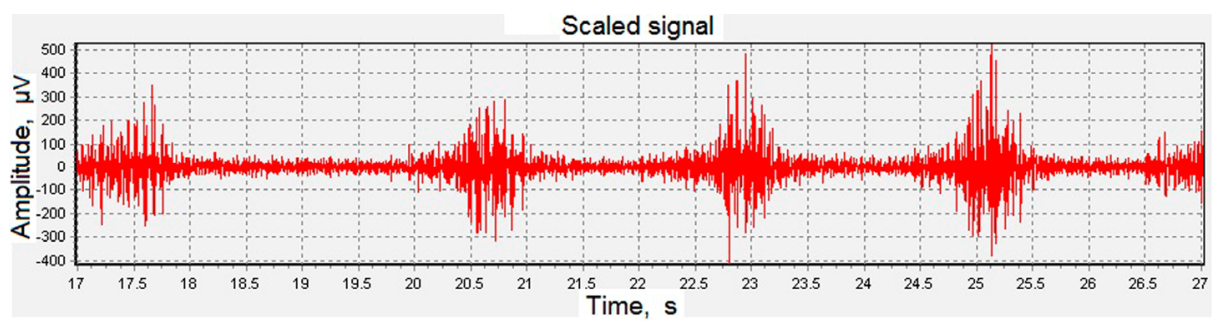

Figure 6. Myographic signals of the point finger activity: $a$ - initial signal; $b$ - filtered signal in the frequency band from 20 to $500 \mathrm{~Hz}$. 
Figure 7 shows miographic signals of the thumb, index and middle finger flexions according to S.A. Vorotnikov.

a)

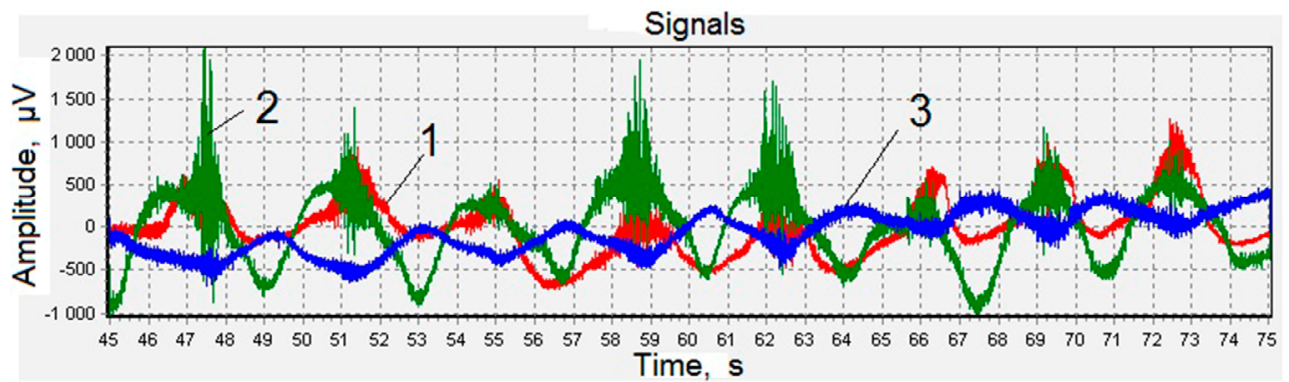

b)
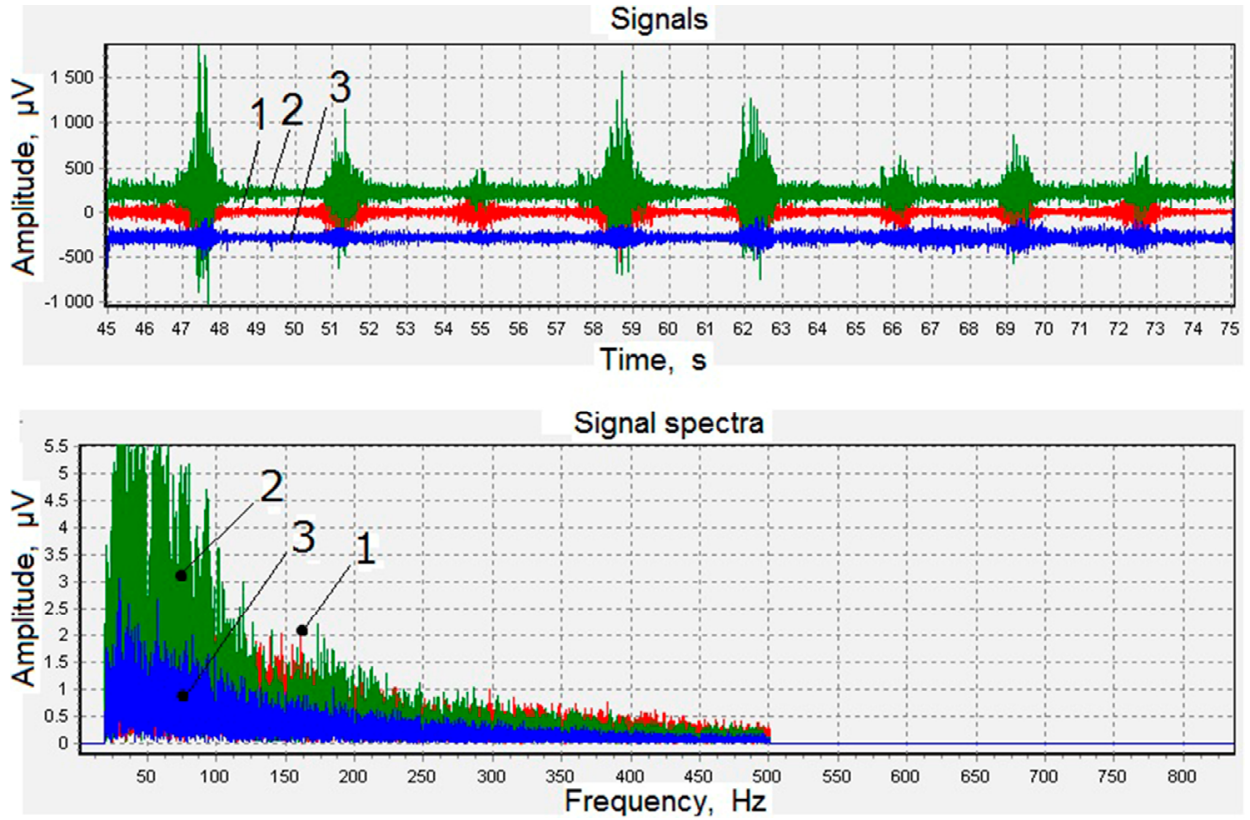

Figure 7. Electromyograms of the finger active flexions: point finger (1), middle finger (2) and a thumb (3): a - in the frequency band from 0 to $10000 \mathrm{~Hz}$; b - in the frequency band from 20 to 500 $\mathrm{Hz}$.

The obtained results showed that filters dramatically distort the waveform, reduce the energy, and remove phase shifts (Figure 7). Since the low-frequency range is not considered, a general picture of electrophysiological processes during muscular contraction cannot be fully evaluated using available modern technologies.

According to the analyzed data, the filter input circuits in bioelectric measuring instruments have a negative impact, thereby reducing the amount of data to be obtained. Modern level of development of the material engineering, computing tools and electrophysiological research potentials exceeds the quality of the current level of myographic research and prosthetic bioelectric control. In addition, the implementation of modern techniques of data processing and obtaining is crucial to provide a general picture of muscle activity. 


\section{Conclusion}

The conducted research confirmed the efficiency of the electrode placement scheme proposed by Vorotnikov for electromyographic study in a wide frequency range using a high-resolution nanosensor-based electromyograph. The obtained electromyograms enabled determination of the patient psychosomatic reaction on changes in visual perception through the example of muscles. The distinguishing features of bioelectric signal enables its utilization for the threshold control in conventional and intelligent prostheses using data recognition patterns.

The input filter circuits limiting the full scale of electromyograph significantly distort the waveform, decrease its energy, and remove phase shifts, thereby reducing the amount of valuable information, especially in the low-frequency range. In addition, the use of filters decelerates signal processing and increases the time response of control system to the input signal. Therefore, it is necessary to find new techniques to measure and process myoelectric signals, which correspond to the current level of technology development, to create new approaches to bioelectric control, and to investigate electrophysiological muscle activity to improve the lives of amputees.

\section{Acknowledgement}

The research was financially supported by the Federal Targeted Program "Research and Development in Priority Fields of S\&T Complex of Russia in 2014-2020", the Agreement No.14.578.21.0032 dated 05.06.2014 "Development of the experimental sample of a hardware-software complex for noninvasive recording of heart micropotentials in a wide frequency band without filtering and averaging in real time to early detect the symptoms of sudden cardiac death", the unique identifier of the contract: RFMEF157814X0032.

\section{References}

[1] Upper extremity prosthetics.

URL:

http://biomed.brown.edu/Courses/BI108/BI108_2003_Groups/Athletic_Prosthetics/ UpperLimb.

[2] E.E. Vanderwerker, Inter-Clinic Information Bulletin 15, 15 (1976)

[3] Forearm external cosmetic prosthesis.

URL: http://www.medicalexpo.com/prod/rslsteeper/product-74956-458446.html

[4] Ch. Cipriani, M. Controzzi, M.Ch. Carrozza, J. of Neuro Engineering and Rehabilitation 8, 29 (2011) doi: 10.1186/1743-0003-8-29

[5] N.V. Turushev, M.G. Grigoriev, D.K. Avdeeva, Modern equipment and technologies: Proceedings of the XX International scientific-practical conference of students, graduate students and young scientists, 361 (2014)

[6] N.V. Turushev, M.G. Grigoriev, D.K. Avdeeva, 2014 the 4th International Workshop on Computer Science and Engineering - Summer, WCSE 2014, 110947(2014)

[7] C.J. De Luca, J. Med. Eng. Technol. 2, 57 (1978) doi: $10.3109 / 03091907809161756$

[8] M. Asghari, H. Hu, Biomedical Signal Processing and Control 2, 275 (2007) doi: 10.1016/j.bspc.2007.07.009

[9] S.A. Vorotnikov, V.S. Strunin, N.A. Vybomov, Caspian Magazine: Management and High Technology (3), 147 (2013) 
[10] N. Jiang, S. Dosen, K. Muller, D. Farina, IEEE Signal Processing Mag. 29(5), 147 (2012) doi: 10.1109/MSP.2012.2203480

[11] M.L. Ivanov, Development and investigation of the ECG hardware-software complex based on nanosensors for recording of heart micropotentials in real time without averaging and filtering, PhD thesis (TPU, Tomsk, 2015)

[12] D.R. Safin, I.S. Pilshicov, M.A. Urakseev, V.G. Gusev, Volga region. Technical sciences (2), 88 (2009) 